\title{
Scattering of halo nuclei on heavy targets at energies around the Coulomb barrier
}

\section{The case of ${ }^{11} \mathrm{Be}$ on ${ }^{197} \mathrm{Au}$}

V. Pesudo ${ }^{1,2, \star}$, M.J.G. Borge ${ }^{3,4}$, A.M. Moro ${ }^{5}$, J.A. Lay ${ }^{5}$, E. Nácher ${ }^{3}$, J. Gómez-Camacho ${ }^{5,6}$, O. Tengblad ${ }^{3}$, L. Acosta $^{7}$, M. Alcorta ${ }^{8}$, M.A.G. Alvarez ${ }^{9}$, C. Andreoiu ${ }^{10}$, P.C. Bender ${ }^{11}$, R. Braid ${ }^{12}$, M. Cubero ${ }^{13}$, A. Di Pietro ${ }^{14}$, J.P. FernándezGarcía $^{5}$, P. Figuera ${ }^{14}$, M. Fisichella ${ }^{14}$, B.R. Fulton ${ }^{15}$, A.B. Garnsworthy ${ }^{11}$, G. Hackman ${ }^{11}$, U. Hager ${ }^{16}$, O.S. Kirsebom $^{17}$, K. Kuhn ${ }^{12}$, M. Lattuada ${ }^{14,18}$, G. Marquínez-Durán ${ }^{19}$, I. Martel $^{19}$, D. Miller ${ }^{11}$, M. Moukaddam ${ }^{11}$, P.D. $\mathrm{O}^{\prime}$ Malley ${ }^{5}$, A. Perea ${ }^{5}$, M.M. Rajabali ${ }^{11}$, A.M. Sánchez-Benítez ${ }^{19}$, F. Sarazin ${ }^{12}$, V. Scuderi ${ }^{14}$, C.E. Svensson ${ }^{20}$, C. Unsworth $^{11}$, and Z.M. Wang ${ }^{10,11}$

${ }^{1}$ Department of Physics, University of the Western Cape, P/B X17, Bellville ZA-7535, South Africa

${ }^{2}$ iThemba LABS, Somerset West 7129, South Africa

${ }^{3}$ Instituto de Estructura de la Materia, CSIC, 28006 Madrid, Spain

${ }^{4}$ ISOLDE-EP, CERN, CH-1211 Geneva 23, Switzerland

${ }^{5}$ Dept de Física Atómica, Molecular y Nucl., Univ. de Sevilla, 41080 Sevilla, Spain

${ }^{6} \mathrm{CN}$ de Aceleradores (U. Sevilla, J. Andalucía, CSIC), 41092, Sevilla, Spain

${ }^{7}$ Instituto de Física. Universidad Nacional Autónoma de México, A.P. 20-364, Cd.Mx. 01000 Mexico

${ }^{8}$ Physics Division, Argonne National Laboratory, Argonne, Illinois 60439, USA

${ }^{9}$ Instituto de Física, Universidade de São Paulo, 05508-090, São Paulo, Brazil

${ }^{10}$ Dept. of Chemistry, Simon Fraser University, Burnaby, BC, Canada V5A 1 S6

${ }^{11}$ TRIUMF, 4004 Wesbrook Mall, Vancouver, British Columbia V6T 2A3, Canada

${ }^{12}$ Physics Department, Colorado School of Mines, Golden, Colorado 80401, USA

${ }^{13}$ CICANUM, Universidad de Costa Rica, Apdo. 2060, San José, Costa Rica

${ }^{14}$ Laboratori Nazionali del Sud, INFN, via Santa Sofia 62, 95123, Catania, Italy

${ }^{15}$ Department of Physics, University of York, YO 10 5DD Heslington, York, UK

${ }^{16}$ National Superconducting Cyclotron Laboratory, Michigan State University, East Lansing, MI 48824, USA

${ }^{17}$ Department of Physics and Astronomy, Aarhus University, 8000 Aarhus, Denmark

${ }^{18}$ Dipart. di Fisica e Astronomia, via Santa Sofia 64, 95123, Catania, Italy

${ }^{19}$ Departamento de Ciencias integradas, Universidad de Huelva, 21071 Huelva, Spain

${ }^{20}$ Dept. of Physics. University of Guelph, Guelph, Ontario N1G 2W1, Canada

\begin{abstract}
This work reports on the scattering of ${ }^{11} \mathrm{Be}$ on ${ }^{197} \mathrm{Au}$ at energies around and below the Coulomb barrier. By experimentally identifying the elastic scattering, inelastic scattering and breakup channels, and comparing them with different calculations, valuable information on the ${ }^{11} \mathrm{Be}$ structure and its $B(E 1)$ distribution to the continuum are obtained. On top of that, a deeper understanding of the scattering process at low energies is achieved for reactions of this kind, making these studies extendable to other loosely-bound systems like ${ }^{17,19} \mathrm{C}$.
\end{abstract}

\section{Introduction}

Low energy nuclear reactions have been a major source of knowledge in the field of nuclear physics since its very inception. With the constant development of new targets and ion sources, more and more nuclei can be produced and accelerated via the ISOL method, so low-energy nuclear reactions can be used to study more exotic nuclei. Hence, experimental techniques and theoretical approaches must be revisited for new data and calculations to continue providing significant contributions to the field.
It is well known that weakly bound nuclei, such as halo nuclei, have a strong $B(E 1)$ to the states in the continuum, which means that undergo breakup relatively easily when exposed to a Coulomb field. This has been thoroughly studied at intermediate and high energies using heavy targets and restricting to very small scattering angles, and the $B(E 1)$ to the continuum of halo nuclei has been extracted [1-4]. These measurements, however, are not straightforward and depending on the structure model and the treatment of the nuclear component, the results can differ significantly in absolute value, like in the case of [2] and [4].

\footnotetext{
^e-mail: vicentepesudo@tlabs.ac.za
} 
This work is part of a series of experiments studying reactions of light halo nuclei on heavy targets at energies close the Coulomb barrier [5-14]. These experiments aim at understanding the dynamics of reactions involving a weakly bound light halo nucleus in a slowly varying intense Coulomb field. The field is felt by the projectile for a long time and adiabatic rearrangements can happen, leaving it in a more favorable configuration to break up and modifying the differential cross sections for the main reaction channels. This work, first, shows the dominance of the Coulomb interaction in this scenario and then validates the method for extracting valuable information on the reaction dynamics and structure properties (such as $B(E 1)$ distributions) of weakly bound nuclei. This property had so far mainly been obtained using high energy beams and this is the first time that the full analysis is consistent with former experiments performed at higher energies $[1,4]$.

The case of ${ }^{11} \mathrm{Be}\left(j^{\pi}=1 / 2^{+}\right.$in its ground state $)$is particularly interesting because it has a $1 / 2^{-}$bound excited state at $E_{x}=320 \mathrm{keV}$ and because the ${ }^{10} \mathrm{Be}$ core is well deformed $\left(\beta_{2}=0.67\right)$. The main reaction channels are, hence, elastic scattering, inelastic scattering and breakup $\left(S_{n}=0.5 \mathrm{MeV}\right.$ [15]). The experimental angular distribution of all of them, compared with different calculations, provides very valuable information on the reaction process and the structure of the ${ }^{11} \mathrm{Be}$ projectile.

\section{Experimental setup}

The experiment was performed at TRIUMF, Vancouver, Canada. The $500 \mathrm{MeV}$ proton beam from the main cyclotron hit a $20 \mathrm{~g} / \mathrm{cm}^{2}$ Ta target in ISAC-II and the ${ }^{11} \mathrm{Be}$ radioactive beam is extracted using the laser ion source TRILIS. Two beam energies were chosen, one around the Coulomb barrier $\left(E_{\mathrm{c} . \mathrm{m} .}=37.1 \mathrm{MeV}, \mathrm{V}_{b}=40\right.$ $\mathrm{MeV})$ and another clearly below the barrier $\left(E_{\mathrm{c} . \mathrm{m} .}=29.6\right.$ $\mathrm{MeV})$. The average current on target was $10^{5} \mathrm{pps}$.

Four silicon $\Delta E-E$ telescopes for the identification of the charged ejectiles were placed inside the scattering chamber (see Fig. 1). Three of them consisted on a 40 $\mu \mathrm{m}$ double-sided silicon strip detectors (DSSSD) backed with a $500 \mu \mathrm{m}$ PAD and the fourth, at backward angles, consisted on a $20 \mu \mathrm{m}$ Single-sided silicon strip detector backed with a $300 \mu \mathrm{m}$ DSSSD. Having a DSSSD in every telescope allowed us to have high granularity and resolution, separating the ${ }^{11} \mathrm{Be}$ from the ${ }^{10} \mathrm{Be}$ fragments after breakup in $\Delta E-E$ two-dimensional plots (see Fig.2). The detectors were directly mounted on a printed circuit board (PCB) and the PCB had been previously cabled to the feedthroughs, reducing the risk of damage of the detectors during the setup.

Around the scattering chamber there were 12 highpurity germanium clovers of TIGRESS, each of them consisting of four eight-fold segmented crystals. The coincidence of the $320 \mathrm{keV}$ gamma ray with ${ }^{11} \mathrm{Be}$ fragment was used to identify the inelastic scattering (Fig. 3), and the

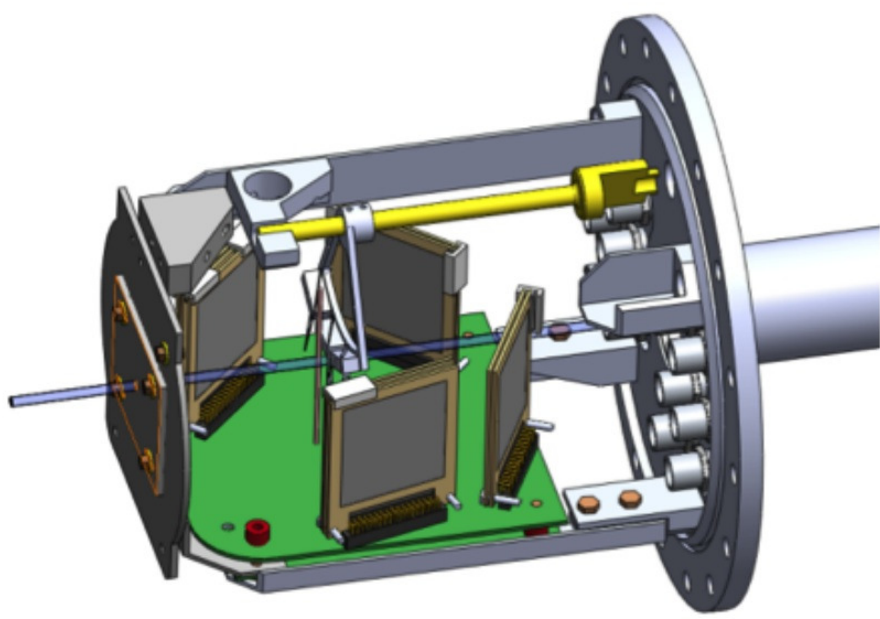

Figure 1. CAD image of the setup, with the four silicon telescopes mounted on a PCB. The pipe on the right hand side of the caption correspond to the upstream side of the chamber.

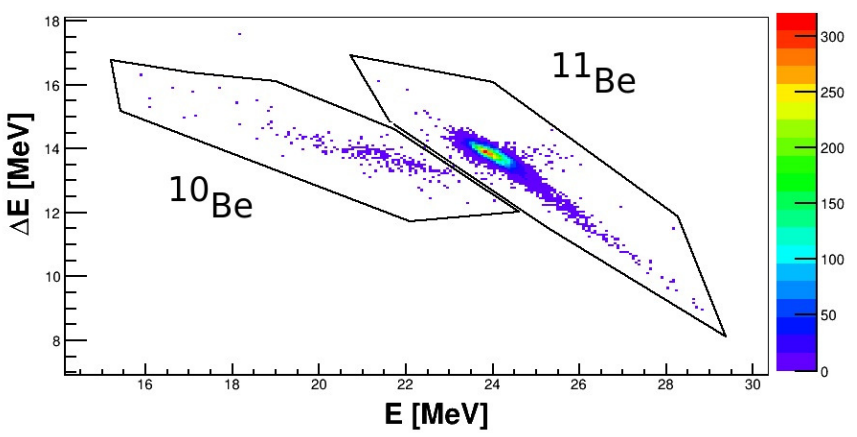

Figure 2. Energy deposited in the back detector in the $x$ axis versus energy deposited in the $\Delta \mathrm{E}$ detector in the $y$ axis. The ${ }^{10} \mathrm{Be}$ and ${ }^{11} \mathrm{Be}$ fragments, corresponding to breakup and quasielastic scattering can be separated.

coincidence with the 279 gamma ray from ${ }^{197} \mathrm{Au}$ for estimating the excitation of the target. The segmentation of the germanium and silicon detectors allowed for a Doppler correction of the $320 \mathrm{keV}$ gamma ray emitted in-flight (Fig. 4).

\section{Theoretical models}

The results are compared with optical model (OM) calculations, first-order semiclassical calculations (the equivalent photon method, EPM, from Ref. [16]), continuum-discretized coupled-channels calculations (CDCC) in which the ${ }^{11} \mathrm{Be}$ was described using singleparticle states of the neutron with respect to the ${ }^{10} \mathrm{Be}$ core, and CDCC including the ${ }^{10} \mathrm{Be}$ core excitation degree of freedom (XCDCC).

The optical model calculation was performed using central Woods-Saxon potentials for both the real and the imaginary parts. In the semiclassical model the projectile is considered to follow a classical Coulomb trajectory and the $E 1$ excitation is treated perturbatively, both for the 


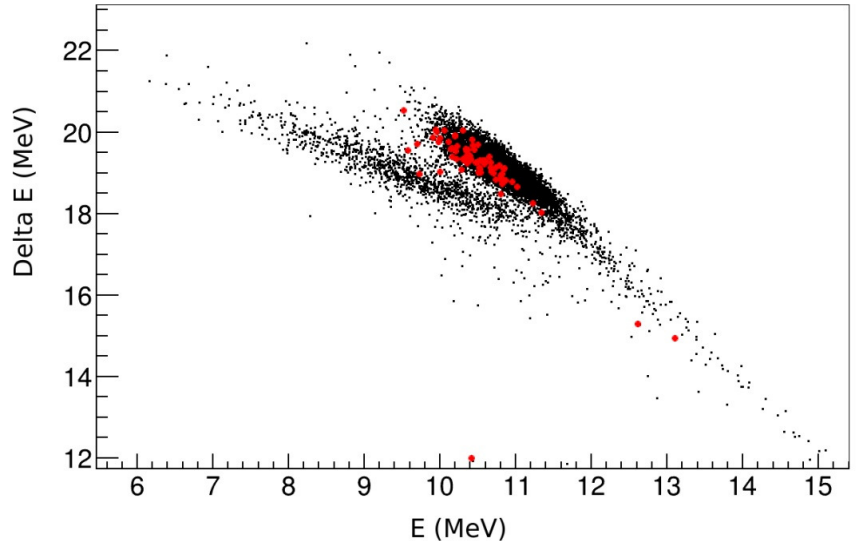

Figure 3. Two dimensional plot with the coincidence with the $320 \mathrm{keV}$ gamma ray depicted in red.

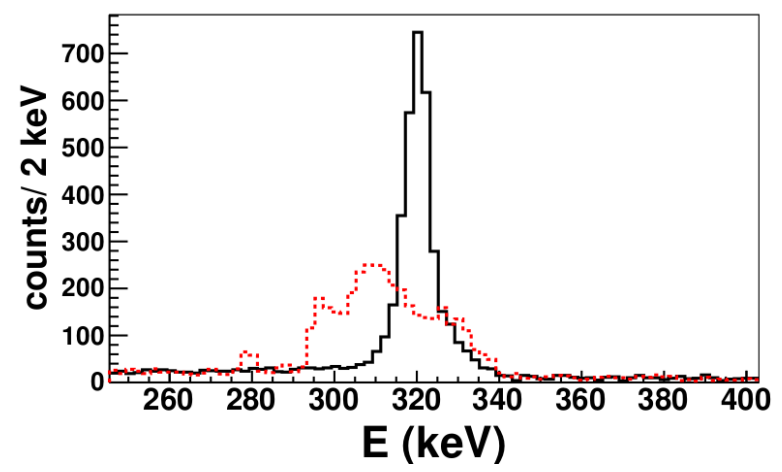

Figure 4. Raw and Doppler-corrected gamma spectrum in coincidence with $\mathrm{T} 1$, the telescope at foremost angles.

inelastic scattering and for the breakup. Since the calculation is performed at first order, it does not account for any rearrangement or adiabatic evolution of the incoming nuclei.

Two CDCC calculations were also performed. The first one uses a single particle (SP) structure model for the ${ }^{11} \mathrm{Be}$, considering only the states of the neutron in a spherical potential of a frozen ${ }^{10} \mathrm{Be}$ core $\left(j^{\pi}=0^{+}\right)$. The second one has an added level of sophistication and takes into account that the neutron can be coupled to the ${ }^{10} \mathrm{Be}$ in its ground state, but also in a $2^{+}$excited state. In the model used (from Ref. [17]), the ${ }^{10} \mathrm{Be}$ ground state has a permanent deformation of $\beta_{2}=0.67$ [18]. The coupling with the $2^{+}$state is included within the rotational model. The ground state, for instance, will be an admixture of $\left|{ }^{10} \mathrm{Be}\left(0^{+}\right) \otimes v\left(s_{1 / 2}\right)\right\rangle_{1 / 2^{+}},\left|{ }^{10} \operatorname{Be}\left(2^{+}\right) \otimes v\left(d_{3 / 2}\right)\right\rangle_{1 / 2^{+}}$and $\left|{ }^{10} \mathrm{Be}\left(2^{+}\right) \otimes v\left(d_{5 / 2}\right)\right\rangle_{1 / 2^{+}}$. It is worth pointing out that the first one is the actual halo configuration, since the centrifugal barrier of the $d$ orbit hinders the development of the halo. For further details on the calculations, see Ref. [19].

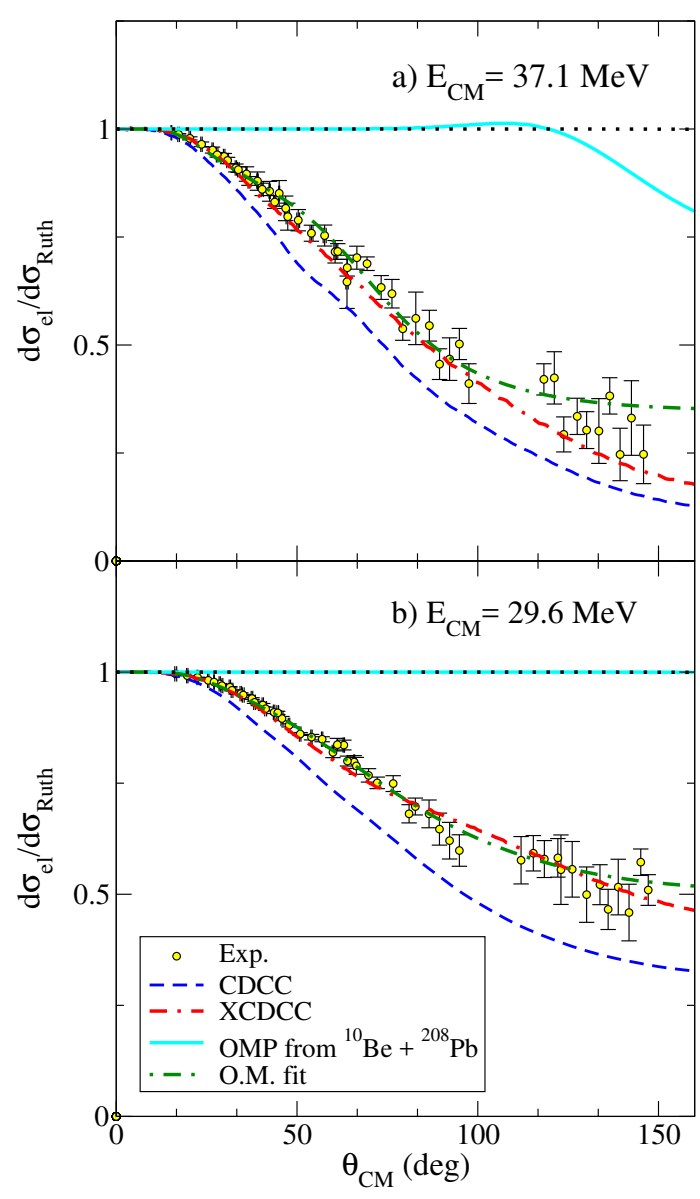

Figure 5. Measured differential elastic scattering cross section at (a) $E_{\mathrm{cm}}=37.10 \mathrm{MeV}$ and (b) $29.64 \mathrm{MeV}$, compared with the CDCC and XCDCC calculations described in the text and within an optical model using central Woods-Saxon potentials.

\section{Discussion}

The first remarkable feature is the deviation of the elastic scattering cross section from Rutherford cross section at very low scattering angles, even at energies well below the barrier. This is an indication of strong dipole polarizability [20] and a pattern often observed in weakly bound nuclei, specially pronounced in halo nuclei $[11,12]$. In order to reproduce this behaviour within the OM, unusually large values for the diffuseness were needed (see table 1). The diffuseness gives an idea of the steepness of the potential and such values are a signature of the presence of long-range couplings.

Also within the OM, a study of the radius of sensitivity was performed. It consisted on fixing the value of the imaginary diffuseness to a certain value, and perform the minimization releasing the rest of parameters. The $\chi^{2}$ of the different minimizations is presented in Fig. 6, and in Fig. 7 the imaginary part of the potentials obtained with the different minimizations are shown. The fact that all these potentials, minimized independently, cross each other at a particular radius evidences that the reaction is specially sensitive to the value of the potential in that point. The sensitivity radius we obtain is $R_{s} \sim 35 \mathrm{fm}$, 
Table 1. Values of the parameters for the real and imaginary part of the nuclear potential and $\chi^{2}$ obtained in the minimization. For comparison, the optical model parameters for the scattering of the core nucleus, ${ }^{10} \mathrm{Be}$, on ${ }^{208} \mathrm{~Pb}$ are also presented.

\begin{tabular}{cccc}
\hline & Core & $E_{c . m .}=$ & $E_{c . m .}=$ \\
& ${ }^{10} \mathrm{Be}+{ }^{208} \mathrm{~Pb}[21]$ & $37.10 \mathrm{MeV}$ & $29.64 \mathrm{MeV}$ \\
\hline & & & \\
$V(\mathrm{MeV})$ & 113. & 14.0 & 9.2 \\
$r_{V}(\mathrm{fm})$ & 1.1 & 1.2 & 1.2 \\
$\boldsymbol{a}_{V}(\mathbf{f m})$ & $\mathbf{0 . 6}$ & $\mathbf{3 . 1}$ & $\mathbf{3 . 8}$ \\
\hline & & & \\
$W(\mathrm{MeV})$ & 169 & 0.21 & 0.179 \\
$r_{W}(\mathrm{fm})$ & 1.196 & 1.2 & 1.2 \\
$\boldsymbol{a}_{W}(\mathbf{f m})$ & $\mathbf{0 . 3 0}$ & $\mathbf{8 . 6 8}$ & $\mathbf{8 . 7 3}$ \\
$\chi^{2}$ & & 1.3 & 1.0 \\
\hline \hline
\end{tabular}

much larger than the sum of the radii of the colliding nuclei: $R\left({ }^{11} \mathrm{Be}\right)+R\left({ }^{197} \mathrm{Au}\right)=7.3 \mathrm{fm}+7.0 \mathrm{fm}=14.3 \mathrm{fm}$. This result is a clear indication of the importance of long range couplings, i.e., of the dominance of the Coulomb interaction.

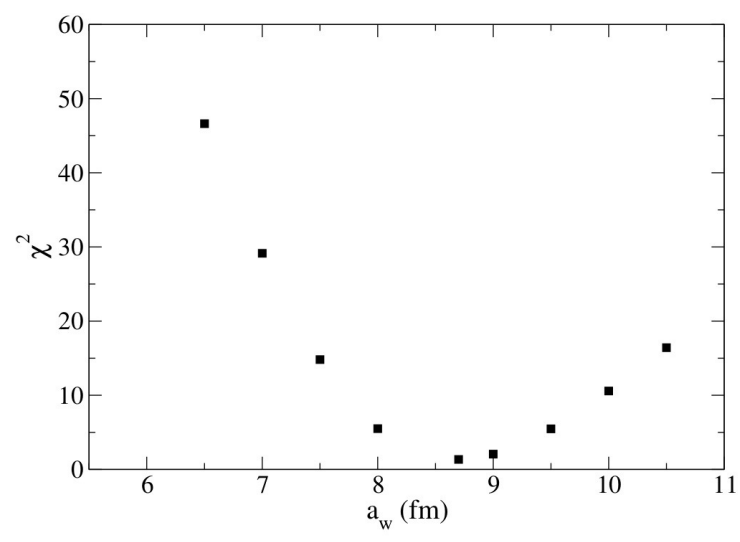

Figure 6. Value of the $\chi^{2}$ obtained for different values of the imaginary diffuseness around the minimum $\left(a_{w}=8.7 \mathrm{fm}\right)$.

For a proper comprehension of the information that the CDCC and conveyed by the XCDCC, it is convenient to discuss the other two reaction channels first. The inelastic scattering and the breakup cross sections are shown in Fig. 8 and Fig. 9, respectively. One can see that the EPM reproduces reasonably well the inelastic scattering cross section at both energies, but fails in reproducing the breakup cross section. Thus, the excitation to the $320 \mathrm{keV}$ bound excited state can be understood as a pure first-order E1 process, while the excitation to the states in the continuum needs of higher-order couplings with higher-order multipolarities.

The case of the CDCC is the opposite, the breakup cross section is reproduced satisfactorily while the inelastic one is not. For the breakup channel to be reproduced, the model space that had to be considered

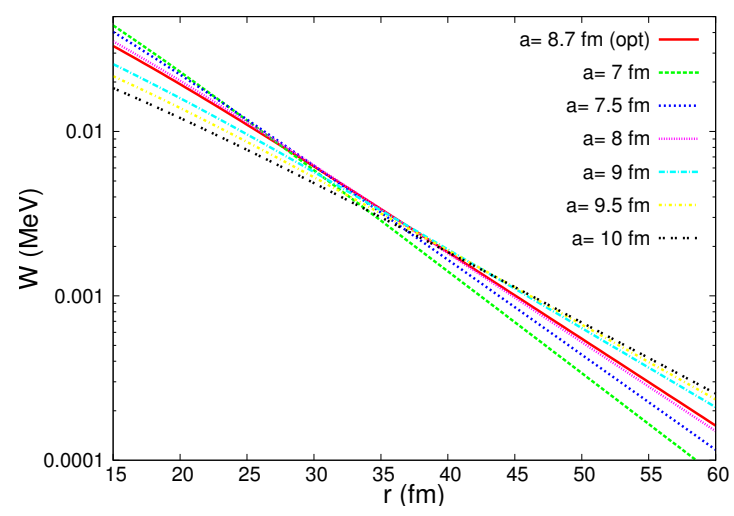

Figure 7. Imaginary part of the potential obtained with the different $\chi^{2}$ minimization shown in Fig. 6. The plot has been zoomed in order to distinguish the crossing point of the different potentials.

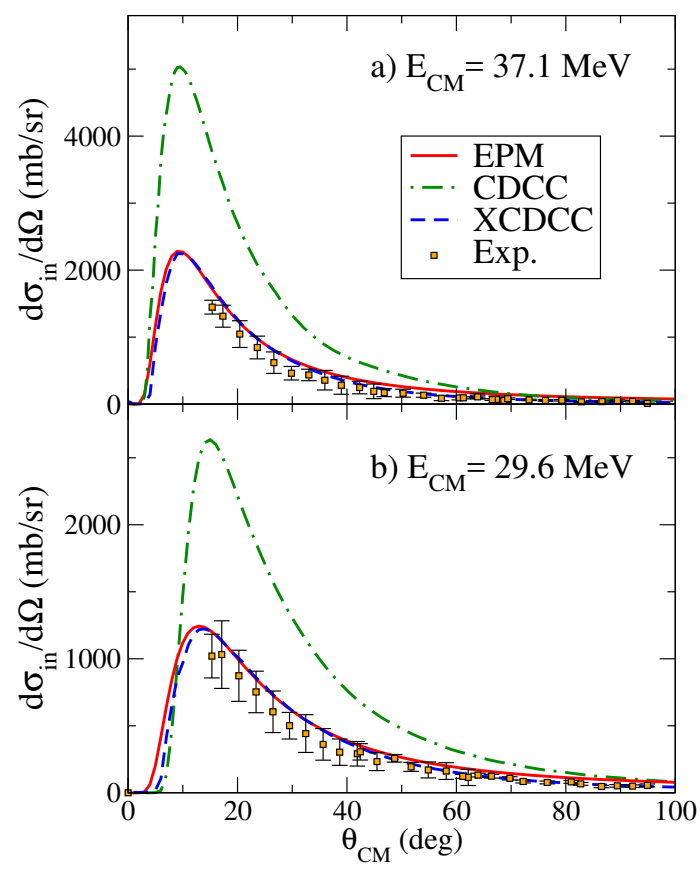

Figure 8. Angular distribution of the inelastic differential cross section of ${ }^{11} \mathrm{Be}+{ }^{197} \mathrm{Au}$, populating the $1 / 2^{-}$bound excited state in ${ }^{11} \mathrm{Be}$ for (a) $E_{\text {c.m. }}=37.10 \mathrm{MeV}$ and (b) $29.64 \mathrm{MeV}$. Experimental data compared with EPM, CDCC and XCDCC calculations described in the text.

in the calculation was large: states in ${ }^{11} \mathrm{Be}$ with $j^{\pi}$ $\leq 15 / 2^{ \pm}$and excitation energies $E_{x} \leq 12 \mathrm{MeV}$ were included. The importance of both, high-order (including continuum-continuum couplings) and high-multipolarity couplings, was apparent. The total angular momentum of the reaction, which is classically related with the impact parameter, that needed to be included in the calculations for obtaining convergence is also large, $J<1200$, which reinforces the conclusions obtained via the study of the radius of sensitivity. Calculations varying the depth of the nuclear potential by a $20 \%$, were also performed, clarifying the dominance of the Coulomb potential. 


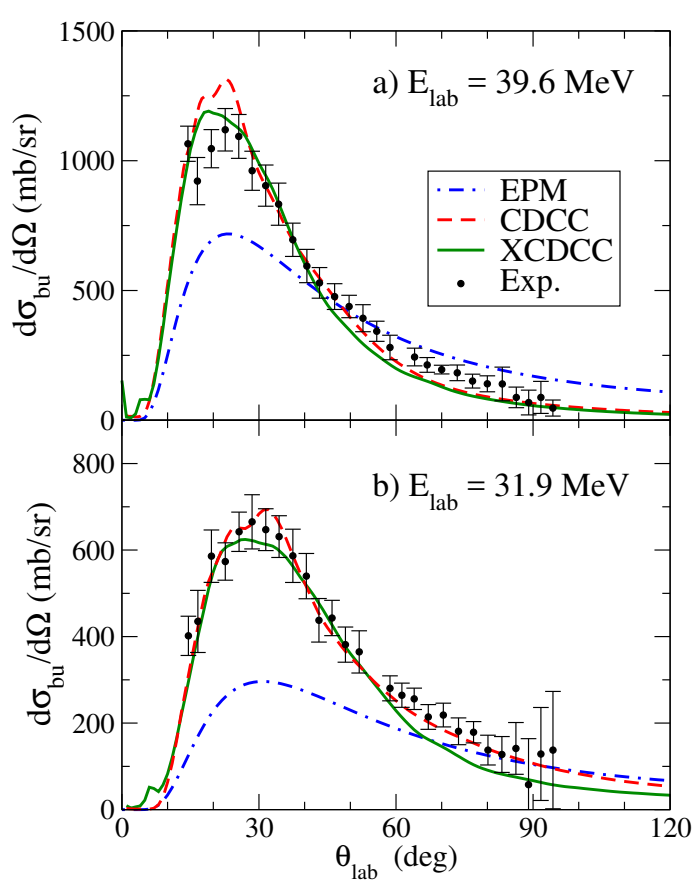

Figure 9. Differential breakup cross section in the laboratory frame at (a) $E_{\text {lab }}=39.6 \mathrm{MeV}$ and (b) $31.9 \mathrm{MeV}$. Experimental data compared with EPM, CDCC and XCDCC calculations described in the text.

On the other hand, the CDCC calculation clearly overestimates the inelastic scattering. This mismatch was expected, since the experimental $B(E 1)$ to the bound excited state $\left(B\left(E 1: 1 / 2^{+} \rightarrow 1 / 2^{-}\right)=0.116 e^{2} \mathrm{fm}^{2}[22]\right)$ is overestimated by a factor of two by the adopted singleparticle model, taken from Ref. [23]. The flux added to this channel is mainly subtracted from the elastic channel, which explains the failure of CDCC in explaining the elastic data. This is not an intrinsic limitation of this parametrization specifically, but of the SP model itself. It is not possible to reproduce the three main outgoing channels: elastic, inelastic and breakup, considering the states of a neutron with respect to a frozen ${ }^{10} \mathrm{Be}$ core. A model that reduces the $B(E 1)$ to the bound excited state, also reduces the $B(E 1)$ to the continuum. In this context the development of the XCDCC formalism was necessary $[24,25]$.

Introducing a more sophisticated model for the ${ }^{11} \mathrm{Be}$ structure, both observables can be reproduced within the same coupled-channels calculation. In particular, we used for the ${ }^{11} \mathrm{Be}$ structure a particle-rotor (PR) model with the parametrization of Ref. [17]. The improvement observed in reproducing the experimental data with respect to the CDCC is because the PR model gives a more realistic $B(E 1)$ than the SP model. The improvement with respect to the EPM is because high-order continuumcontinuum couplings are included in the coupled-channels calculations.

The slight overestimation in the inelastic cross section by both the XCDCC and the EPM reinforces the value for the $B\left(E 1: 1 / 2^{+} \rightarrow 1 / 2^{-}\right)=0.102 \mathrm{e}^{2} \mathrm{fm}^{2}$ obtained by Kwan et al. in Ref. [26] over the previous value of $B(E 1)=0.116 \mathrm{e}^{2} \mathrm{fm}^{2}$ obtained by Millener et al. in Ref. [22] and used in these calculations.

Fig. 10 shows the $B(E 1)$ extracted from previous works at higher energies (Refs. [2, 4]) and the one extracted from the structure models used in this work. The model from Ref. [17] that explains all our observables, reproduces the $B(E 1)$ obtained by Fukuda et al. in Ref. [4]. The data in Ref. [2], despite following the same pattern, differs in absolute magnitude, so this work has the added value of disentangling this incompatibility. Another interesting feature is that the $3.37 \mathrm{MeV}$ gamma ray from the decay of the $2^{+}$to the $0^{+}$ground state in ${ }^{10} \mathrm{Be}$ was not observed. Although there is a non-negligible contribution of states above the energy of the $2^{+}$, the average excitation energy is around $1 \mathrm{MeV}$ (well below the $3.87 \mathrm{MeV}$ that would be needed for this decay channel to be open). This observation, however, is consistent with the nucleus being predominantly broken up from its halo configuration, with the neutron coupled to the ${ }^{10} \mathrm{Be}$ ground state.

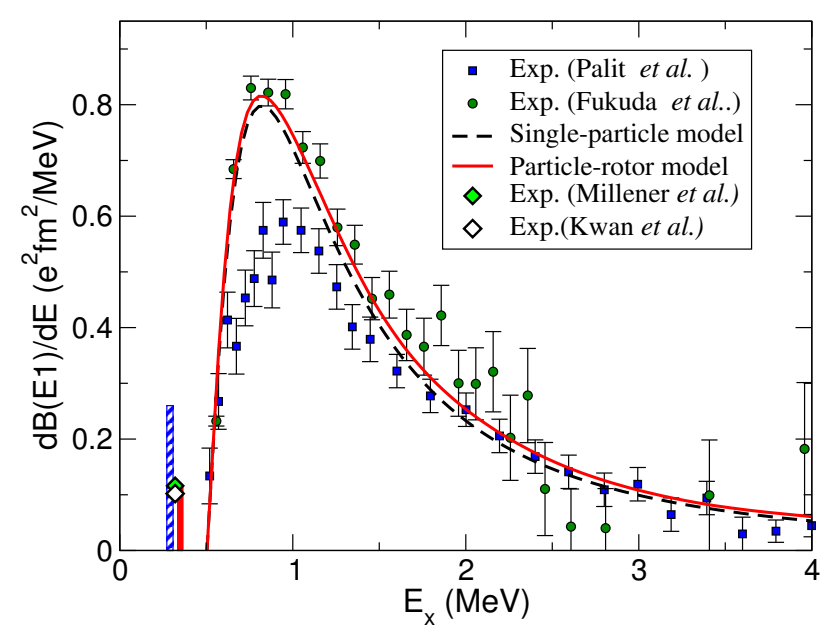

Figure 10. $B(E 1)$ strength distributions for ${ }^{11} \mathrm{Be}$. The experimental data in the continuum are from the Coulomb dissociation experiments of Refs. [2, 4]. The filled diamond represent the experimental $B\left(E 1 ; 1 / 2^{+} \rightarrow 1 / 2^{-}\right)$value $\left(\right.$in $\left.\mathrm{e}^{2} \mathrm{fm}^{2}\right)$ between the bound states of ${ }^{11} \mathrm{Be}[22,26]$ and the vertical bars the output of the calculation. The theoretical distributions correspond to the SP and PR models.

\section{Conclusions}

The three main reaction channels, namely, elastic scattering, inelastic scattering and breakup have been measured and separated at energies around and below the Coulomb barrier for the ${ }^{11} \mathrm{Be}+{ }^{197} \mathrm{Au}$ reaction. The dominance of the Coulomb interaction in this regime over most of the angular range has been inferred independently via optical model and CDCC calculations. The XCDCC calculation reproduces all the observables simultaneously and, together with the experimental procedure presented here, is 
a powerful tool to understand the dynamics of the reaction and are complementary to high-energy Coulomb breakup measurements for determining structure properties of the weakly bound nuclei involved, such as the $B(E 1)$. The $B(E 1)$ obtained with Tarutina's model in Ref. [17] reproduces our data and the data measured in RIKEN at intermediate energies, validating these results.

The reaction dynamics of the scattering process are strongly determined by the slowness of the collision, during which the weakly bound nucleus has time to evolve adiabatically into configurations from which breakup is specially favorable, enhancing this cross section with respect to the pure first-order direct breakup.

\section{Acknowledgements}

This work was supported by the Spanish Government under the projects FPA2015-64969-P, FIS2014-53448C2-1-P, FPA2013-47327-c02-01-R, FPA2012-32443, FIS2013-41994-P and Consolider CPAN CSD200700042; the EUROCORES project EUI-2009-04162; the European Unions Horizon 2020 research and innovation program under grant agreement No 654002; the Helmholtz Association (HGT) through the Nuclear Astrophysics Virtual Institute (VH-VI-417); ATI Sistemas; The Natural Sciences and Engineering Research Council of Canada; The Canada Foundation for Innovation; and the British Columbia Knowledge Development Fund. TRIUMF receives federal funding via a contribution agreement through the National Research Council of Canada. OSK acknowledges support from the Villum Foundation.

\section{References}

[1] T. Nakamura et al., Phys. Lett. B 331B, 296 (1994)
[2] R. Palit et al., Phys. Rev. C 68, 034318 (2003)

[3] T. Nakamura et al., Phys. Rev. Lett. 96, 252502 (2006)

[4] N. Fukuda et al., Phys. Rev. C 70, 054606 (2004)

[5] M. Mazzocco et al., Eur. Phys. J. A 28, 295 (2006)

[6] D. Escrig et al., Nucl. Phys. A 792, 2 (2007)

[7] A. M. Sánchez-Benítez et al., Nucl. Phys. A 803, 30 (2008)

[8] L. Acosta et al., Eur. Phys. J. A 42, 461 (2009)

[9] L. Acosta et al., Phys. Rev. C 84, 044604 (2011)

[10] A. Di Pietro, et al., Phys. Rev. Lett. 105, 22701 (2010)

[11] M. Cubero, et al., Phys. Rev. Lett. 109, 262701 (2012)

[12] A. Di Pietro, et al., Phys. Rev. C 85, 054607 (2012)

[13] J. P. Fernández-García et al., Phys. Rev. Lett. 110, 142701 (2013)

[14] J. P. Fernández-García et al., Phys. Rev. C 92, 044608 (2015)

[15] J.H. Kelley et al., Nucl. Phys. A 88, 880 (2012)

[16] K. Alder, A. Winther, Electromagnetic excitation (North Holland, Amsterdam, 1975)

[17] T. Tarutina, L.C. Chamon, M.S. Hussein, Phys. Rev. C 67, 044605 (2003)

[18] F.M. Nunes et al., Nucl. Phys. A 609, 43 (1996)

[19] V. Pesudo et al., Phys. Rev. Lett. 118, 152502 (2017)

[20] M. V. Andres, J. Gomez-Camacho, Phys. Rev. Lett. 82, 001387 (1999)

[21] J.J. Kolata et al., Phys. Rev. C 69, 047601 (2004)

[22] D.J. Millener, J.W. Olness, E.K. Warburton, S.S. Hanna, Phys. Rev. C 28, 497 (1983)

[23] P. Capel, G. Goldstein, D. Baye, Phys. Rev. C 70, 064605 (2004)

[24] N.C. Summers, F.M. Nunes, I.J. Thompson, Phys. Rev. C 73, 031603 (2006)

[25] R. de Diego, J.M. Arias, J.A. Lay, A.M. Moro, Phys. Rev. C 89, 064609 (2014)

[26] E. Kwan et al., Phys. Lett. B 732, 210 (2014) 